\title{
Structure-driven remanent high-spin state in metallosupramolecular assemblies
}

\author{
Y. Bodenthin, ${ }^{1,2}$ G. Schwarz, ${ }^{3}$ Z. Tomkowicz, ${ }^{4}$ A. Nefedov, ${ }^{5}$ M. Lommel, ${ }^{1}$ H. Möhwald, ${ }^{3}$ W. Haase, ${ }^{6}$ D. G. Kurth,${ }^{3,7}$ and \\ U. Pietsch ${ }^{1}$ \\ ${ }^{1}$ University of Siegen, FB7 Solid State Physics, D-57068 Siegen, Germany \\ ${ }^{2}$ Swiss Light Source, Paul Scherrer Institut, CH-5232 Villigen-PSI, Switzerland \\ ${ }^{3}$ Max Planck Institute of Colloids and Interfaces, D-14424 Potsdam, Germany \\ ${ }^{4}$ Jagellonian University, Institute of Physics, Reymonta 4, 30-059 Krakow, Poland \\ ${ }^{5}$ Ruhr-University Bochum, Institute of Experimental Physics and Solid State Physics, D-44780 Bochum, Germany \\ ${ }^{6}$ Darmstadt University of Technology, Institute of Physical Chemistry, Petersenstrasse 20, D-64287, Germany \\ ${ }^{7}$ National Institute for Materials Science, 1-1 Namiki, Tsukuba, Ibaraki 305-0044, Japan
}

(Received 11 March 2006; revised manuscript received 7 February 2007; published 16 August 2007)

\begin{abstract}
A versatile approach designing supramolecular assemblies for molecular magnetism relies on metallosupramolecular polyelectrolyte amphiphile complexes, which are self-assembled from ditopic bisterpyridine ligands, transition metal ions, and amphiphilic molecules. We report on measurements at powdered samples using energy-dispersive small angle x-ray scattering, x-ray magnetic circular dichroism, and magnetic measurements. We observe a spin crossover of the $\mathrm{Fe}^{2+}$ ions into a remanent state above room temperature driven and stabilized by a structural phase transition of the two dimensional amphiphilic matrix of the supramolecular complex. The temperature of spin crossover scales with the number of amphiphilic molecules attached to the polyelectrolyte backbone. The spin crossover itself can be rationalized by a transition of the $\mathrm{Fe}^{2+}$ ions from a $S=0, t_{2 g}^{6} e_{g}^{0}$ low-spin state to a magnetic $S=2, t_{2 g}^{4} e_{g}^{2}$ high-spin state and is independent from the number of amphiphiles per unit. The remanence in powdered samples might be caused by the disorder and interdigitation of amphiphilic molecules. Low temperature measurements suggest a possible antiferromagnetic coupling between $\mathrm{Fe}^{2+}$ ions.
\end{abstract}

DOI: 10.1103/PhysRevB.76.064422

PACS number(s): 75.50.Xx, 75.75.+a, 81.16.Fg, 81.16.Dn

\section{INTRODUCTION}

Molecular magnetism represents a very lively scientific area where both physicists and chemists join their efforts to design molecular architectures containing magnetic metal centers with novel magnetic properties. ${ }^{1-3}$ In contrast to the current line of molecular magnetism to synthesize molecules or molecule clusters with large spins, the concept of designing supramolecular assemblies by self-assembly of ditopic ligands, transition metal ions, and amphiphilic molecules is most flexible and tunable. Such materials exhibit fundamental features such as magnetic and optical transitions and bistability that make them attractive for further investigations and applications such as storage media, signal processing ${ }^{4}$ and transduction ${ }^{5}$ or new theoretical aspects like testing the validity of quantum mechanical approaches at nano or mesoscopic length scales. ${ }^{6}$ Combining both strategies and understanding the design principles in order to tailor magnetic functions in these supramolecular systems is therefore a central objective in physics, supramolecular chemistry and materials science. One possible route of research is the synthesis of large molecules or clusters providing intrinsic magnetism. ${ }^{7}$ These systems often contain one or more transition metal ions with a $3 d^{n} \quad(4 \leq n \leq 7)$ electronic configuration. ${ }^{8}$ If the metal ion is situated in a ligand field of suitable symmetry and strength, the degeneracy of the $d$-orbital energy states is lifted; in case of an octahedral coordination geometry the $d$-states split into $t_{2 g}$ and $e_{g}$ sublevel. ${ }^{9}$ For instance, the nonmagnetic $t_{2 g}^{6} e_{g}^{0}$ population (low-spin state, LS) of $\mathrm{Fe}^{2+}$ is more favorable if the energy separation of the sublevel, the crystal field splitting energy $\Delta$ is larger than the spin pairing energy $P$. Two principle strategies have been adopted in order to affect the spin state of the coordinated metal ion. The strength of the ligand field can be reduced by either steric strain or steric repulsion, thus, making the magnetic high-spin (HS) $t_{2 g}^{4} e_{g}^{2}$ electronic configuration more favorable. ${ }^{10}$ In the first case, the coordination geometry is strained through steric interactions, e.g., through packing effects of counter ions or ligand structure; in the second case, a sterically demanding substituent is introduced close the donor atom such that the metal-donor atom distances are longer than in the unsubstituted ligand. Spin transitions and therewith magnetic transitions in these molecules can be induced thermally if $|\Delta-P| \sim k_{B} T$ and occur in liquids, liquid crystals, and solids. ${ }^{11,12}$ Examples of thermal, light and pressure controlled spin transitions in systems using terpyridine or related ligands is, for instance, described by Breuning et al. in $[2 \times 2] \mathrm{Fe}^{2+}$ grids. ${ }^{13}$ Subsidiary triggering spin transition and magnetic properties by different ligand substituents in such grid systems is shown by Ruben et al. and Waldmann et al. ${ }^{14,15}$ Nevertheless, in these systems none or only weak intermolecular interactions between the spin transition molecules occur and the molecules are affected directly by light, temperature, pressure, etc.

In order to extend the given concepts to induce spin transitions, we have developed another route. The spin transition or spin crossover is induced by deliberately distorting the coordination geometry through an amphiphilic matrix, which embeds a spin crossover polymer. Perturbing the coordination geometry changes the interaction between the ligands and the metal ions thus reducing the ligand field splitting $\Delta$, giving rise to a transition of the $\mathrm{Fe}^{2+}$ ions from a low-spin to 


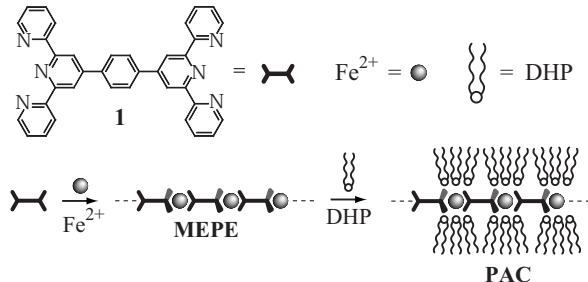

FIG. 1. Self-assembly of ditopic bisterpyridine ligands, $\mathrm{Fe}^{2+}$ ions, and amphiphiles results in metallosupramolecular coordination polyelectrolytes (MEPE) and the corresponding polyelectrolyte-amphiphile complexes (PAC). The ratio of metal centers to DHP is readily adjusted by the assembly conditions. The scheme shows the 1:6 complex.

a high-spin electronic configuration. We recently demonstrated this concept in thin films of metallosupramolecular polyelectrolyte-amphiphile complexes (PAC) based on ditopic bisterpyridines and $\mathrm{Fe}^{2+}$ as central metal ions as schematically shown in Fig. 1. ${ }^{16}$ Melting the amphiphilic matrix induces sufficient mechanical strain to distort the octahedral coordination geometry of the tightly coupled $\mathrm{Fe}^{2+}$ ions in the metallosupramolecular polyelectrolyte backbone. The distortion is strong enough to reduce the strength of the ligand field, giving rise to a spin crossover from low-spin to a highspin state resulting in a diamagnetic to paramagnetic transition.

We note that thermal or optical induced spin transition generally is a molecular process, driven by the entropy gain on going from the LS to the HS state. ${ }^{17}$ Usually, temperature driven spin transition phenomena are classified into a discontinuous type, in which the spin state conversion is accompanied by a thermal hysteresis and into a continuous type, were the conversion takes place without a hysteresis. In many cases, the discontinuous type is accompanied by a structural change and can be described in terms of Landau theory. ${ }^{18}$ As an example, a large thermal hysteresis of $115 \mathrm{~K}$ around room temperature is observed in $\mathrm{Fe}^{3+}\left[\mathrm{Fe}(\mathrm{qsal})_{2}\right] \mathrm{NCSe} 2 \mathrm{DMSO}$ [N-(8-quinolyl)-salicyladimine $].{ }^{19} \mathrm{~A}$ metastable high-spin state at low temperatures in a metal-organic polymer is predicted in terms of generalization of the Slichter-Drickamer regular solution model by introducing interacting spinlike domains. $^{20,21}$ In these thermodynamic models, structural phase transitions take place as consequence of a spin transition. Here, we describe the transition of $\mathrm{Fe}^{2+}$ ions from a low-spin state into a high-spin state as a consequence of a structural transition of the system. In order to distinguish between the well-known entropy driven spin transition and the mechanism described in this article, we use the terminology of spin crossover for the latter one.

\section{RESULTS AND DISCUSSION}

In this work, we show that the $\mathrm{Fe}^{2+}$ ions in PAC in the bulk phase undergo a transition from the nonmagnetic $S=0$, $t_{2 g}^{6} e_{g}^{0}$ low-spin state to a magnetic $S=2, t_{2 g}^{4} e_{g}^{2}$ high-spin state induced by a structural order-disorder transition upon heating. Contrary to thin films that show a reversible but partial spin crossover, in the bulk phase the spin crossover is close to complete and irreversible. The high-spin phase is always stable down to low temperatures of a few Kelvin. We show that the spin crossover temperature $T_{\mathrm{SC}}$ depends on the ratio of metal ions to amphiphiles. We present a detailed analysis of the complex investigated by magnetic measurements, temperature resolved $\mathrm{x}$-ray scattering experiments and $\mathrm{x}$-ray magnetic circular dichroism. In order to prove our model of a structurally induced and stabilized high-spin state we investigate a PAC where the $\mathrm{Fe}^{2+}$ ions are replaced by $\mathrm{Ni}^{2+}$ ions. The $\mathrm{Ni}^{2+}-\mathrm{PAC}$ is isostructural to the $\mathrm{Fe}^{2+}-\mathrm{PAC}$ but is characterized by the absence of a spin transition.

\section{A. Sample preparation and precharacterization}

Figure 1 shows a scheme of the polyelectrolyteamphiphile-complex (PAC), which is made by sequential self-assembly of the ditopic bis-terpyridine ligands 1,4bis $\left(2,2 \prime: 6 \prime, 2\right.$ '-terpyridine-4ı-yl)benzene 1 and $\mathrm{Fe}^{2+}$ ions leading to a metallopolyelectrolyte (MEPE). Self-assembly of MEPE and dihexadecyl phosphate (DHP) as amphiphilic component results in the polyelectrolyte-amphiphilecomplex (PAC). ${ }^{22}$ The ratio of metal centers to amphiphiles is readily adjusted by the self-assembly conditions. Here, we present results for the 1:2, 1:4 and 1:6-complex. In the solid state, PACs show thermotropic polymorphism and the structure of the system can be described by a lateral arrangement of lamella of MEPE separated by hexagonally closed packed DHP strata. ${ }^{23}$ The thermal stability of the complex is proven by differential scanning calorimetry (DSC) and thermogravimetric measurements. There is no evidence for oxidation or decomposition of the sample in a temperature range of $4 \mathrm{~K}$ $\leq T \leq 475 \mathrm{~K}$. For physical investigation, the material is prepared as fine powder containing self-organized domains of random orientation.

\section{B. Magnetic characterization}

The magnetic properties are probed under He-gas atmosphere by a Faraday balance providing a sensitivity of $10^{-6} \mathrm{emu}$ in an external field of $B=1.2 \mathrm{~T} .{ }^{24}$ We use the same heating rate of $2 \mathrm{~K} / \mathrm{min}$ as for the thermal and structural characterization. In order to correct diamagnetic contributions, the molar susceptibility of the amphiphiles is measured leading to a value of $\chi_{M}=-6.0 \pm 0.1 \times 10^{-4} \mathrm{emu} / \mathrm{mol}$ while the susceptibility of the ligand is calculated from tabulated values $\left(\chi_{M}=-3.5 \pm 0.1 \times 10^{-4} \mathrm{emu} / \mathrm{mol}\right) .^{25}$

In Fig. 2 we present the effective magnetic moment $\mu_{\text {eff }}(T)$ in terms of Bohr magnetons per iron ion $\left(\mu_{B} / \mathrm{Fe}^{2+}\right)$ as function of temperature for the 1:2 (red) and the 1:6-complex (black). An increase of the magnetic moment to values of about $\mu_{\text {eff }}=4.8 \mu_{B} / \mathrm{Fe}^{2+}$ indicates that almost all $\mathrm{Fe}^{2+}$ ions of the sample have performed a transition from the $t_{2 g}^{6} e_{g}^{0}, S=0$ low-spin state into the $t_{2 g}^{4} e_{g}^{2}, S=2$ high-spin state. Assuming pure spin magnetism or a complete quenching of orbital contributions, the magnetic moment of free $\mathrm{Fe}^{2+}$ is estimated to be $4.9 \mu_{B} / \mathrm{Fe}^{2+}$. Because deviations from an ideal octahedral symmetry are present in both HS and LS state, this value can be considered as a lower limit. For comparison, Constable et al. report a value of $5.3 \mu_{B} / \mathrm{Fe}^{2+}$ for $\mathrm{Fe}^{2+}(\mathrm{tpy})_{2}$ complexes 


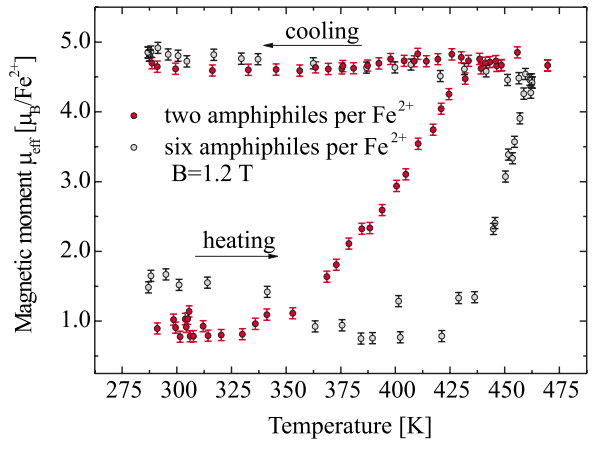

FIG. 2. (Color online) Magnetic moment $\mu_{\mathrm{eff}}(T)$ of two PACs containing different amounts of amphiphilic molecules. The increase of the moment with temperature indicates a transition from diamagnetic $t_{2 g}^{6} e_{g}^{0}, S=0$ low-spin state to a paramagnetic $t_{2 g}^{4} e_{g}^{2}, S$ $=2$ high-spin state of the $\mathrm{Fe}^{2+}$ ions. The spin crossover temperature as well as the gradient of $\mu_{\text {eff }}(T)$ increases with a higher amount of amphiphiles per metal center within the complex. The high-spin state persists upon cooling in all three systems.

with substituents in the $6 \prime$ and $6 \prime \prime$ position of the ligand. ${ }^{10}$ These substituents, as described in the Introduction, reduce the ligand field by steric repulsion leading to not quenched orbital contributions and a $g$ factor of 2.16.

The onset of the spin crossover is estimated to be $363 \mathrm{~K}$ for the complex containing two amphiphiles per metal center. In an interval of $80 \mathrm{~K}$ the complex reaches the saturated high-spin fraction at $443 \mathrm{~K}$. During further heating, the magnetic moment remains constant. The spin crossover temperature at half of the saturated high-spin fraction is estimated to be $T_{\mathrm{SC}}=410 \mathrm{~K}$. The high-spin state remains stable during cooling down to room temperature even down to a temperature of $4.4 \mathrm{~K}$. Further heating and cooling cycles do not change the spin state of the $\mathrm{Fe}^{2+}$ ions.

The black symbols in Fig. 2 show $\mu_{\mathrm{eff}}(T)$ for the complex containing six amphiphiles per metal center. The initial magnetization at room temperature corresponds to a magnetic moment of $\mu_{\text {in }}=1.5 \mu_{B} / \mathrm{Fe}^{2+}$. Slightly above room temperature, the initial magnetization decreases to $0.5 \mu_{B} / \mathrm{Fe}^{2+}$. The residual magnetization might be related to kinks or defects naturally occurring at chain ends in the metallopolyelectrolyte backbone. The absence of a residual magnetization in the complex with only two amphiphiles may be associated with a better structural order of this sample after the preparation. The minimum in the curve coincides with the melting of the amphiphiles at $325 \mathrm{~K}$, which anneal the structure and decrease the number of defects. The spin crossover in the 1:6 complex begins to occur at approximately $438 \mathrm{~K}$. The spin crossover temperature is estimated to be $T_{\mathrm{SC}}=452 \mathrm{~K}$ and is higher than that of the 1:2-complex. The high-spin state with a similar magnetic moment as mentioned above is reached in an interval of $32 \mathrm{~K}$ at $460 \mathrm{~K}$. The high-spin state also persists during cooling. The spin crossover of PAC with four amphiphiles per metal center is estimated to be $T_{\mathrm{SC}}=425 \mathrm{~K}$ and lies in between the 1:2-system with $410 \mathrm{~K}$ and the 1:6system with $452 \mathrm{~K}$ (data not shown). These findings reveal that the temperature of structural changes and therewith the spin crossover temperature $T_{\mathrm{SC}}$ as well as the gradient of $\mu_{\text {eff }}(T)$ increases with the number of amphiphiles per metal center.

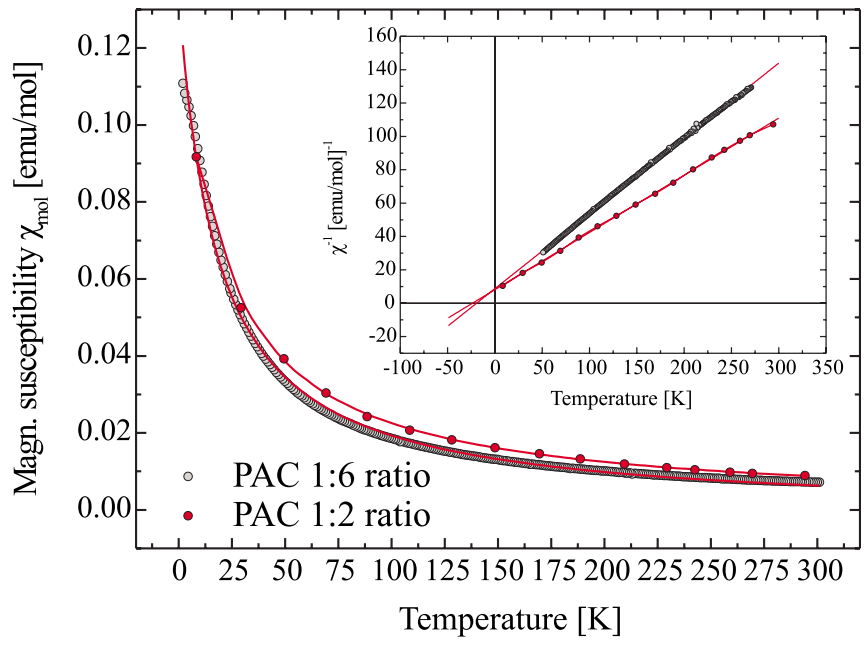

FIG. 3. (Color online) Fitting of the magnetic susceptibility of PACs containing different amounts of amphiphilic molecules to a Curie-Weiss law. The data were taken using a SQUID magnetometer after thermal treatment of the samples to switch the $\mathrm{Fe}^{2+}$ ions into the stable high-spin state. We determine a magnetic moment of $4.8 \pm 0.2 \mu_{B} / \mathrm{Fe}^{2+}$ for both systems and negative Weiss constants of $\Theta=-21.07 \pm 0.56 \mathrm{~K}$ for the $1: 2$ complex (red circles) and $\Theta$ $=-17.99 \pm 0.26 \mathrm{~K}$ for the 1:6 complex (black circles). The inset shows $\chi^{-1}(T)$.

After heating, we cool down the samples to low temperatures of few Kelvin while measuring the magnetic moment with a SQUID magnetometer (Fig. 3). Fitting the data to the Curie-Weiss law

$$
\chi=\frac{C}{T-\Theta} \quad \text { with } C=\frac{N_{A} \mu_{\mathrm{eff}}}{3 k_{B}}
$$

we determine independently the magnetic moment to be $4.8 \pm 0.2 \mu_{B} / \mathrm{Fe}^{2+}$ for both systems which fairly agrees to the values determined with the Faraday balance. We found a negative Weiss constant of $\Theta=-21.07 \pm 0.56 \mathrm{~K}$ for the $1: 2$ complex and $\Theta=-17.99 \pm 0.26 \mathrm{~K}$ for the $1: 6$-complex indicating a possible antiferromagnetic coupling between the $\mathrm{Fe}^{2+}$ centers at very low temperatures. Additionally, these measurements confirm the persistence of the stable high-spin state of the $\mathrm{Fe}^{2+}$ ions down to low temperatures and debilitate the possibility of uncomplete thermal hysteresis of the spin crossover because of a restricted temperature range.

\section{Structural investigations}

To explore the origin of the apparently stable high-spin fraction, we took a detailed look at the structure of PAC at the Energy Dispersive Reflectometer (EDR) at the BESSY II synchrotron. ${ }^{26}$ Here, the hard $\mathrm{x}$-ray decay of a bending magnet is used for scattering providing $x$ rays in an energy range of $5 \mathrm{keV} \leq E \leq 25 \mathrm{keV}$. Small angle $x$-ray scattering (SAXS) patterns are collected as a function of temperature using two energy-dispersive detectors with an energy resolution of $\Delta E / E=10^{-2} .{ }^{27}$ The momentum transfer $q$ is given by 


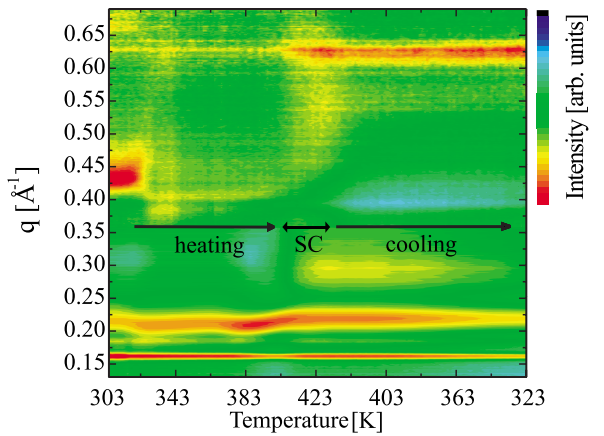

FIG. 4. (Color online) Energy-dispersive SAXS pattern of $\mathrm{Fe}^{2+}$-PAC. The time resolution of $60 \mathrm{~s}$ per spectra allows for determining structural changes as a function of temperature. Clearly, the spin crossover (SC) is connected to the appearance of the new structure, which remains stable during cooling to room temperature.

$$
q=\frac{2 \pi E}{h \cdot c}\left(\sin \alpha_{i}+\sin \alpha_{f}\right)=q \simeq \theta E,
$$

where in the latter equation the glancing angle $\theta$ is given in radiant and the energy in $\mathrm{keV}$. To enhance the accessible $q$ range the detectors are equipped at two fix exit angles of $2 \theta=2.79^{\circ}$ and $2 \theta=5.78^{\circ}$ with respect to the incident beam. This geometry provides good counting statistics in a $q$ range of $0.05 \AA^{-1} \leq q \leq 1.0 \AA^{-1}$. To increase the signal to noise ratio an evacuated flight-tube is placed between the sample and the detectors. This setup provides a time resolution of $60 \mathrm{~s}$ per spectrum and allows determining structural changes of the complex as function of temperature.

Figure 4 summarizes the collected SAXS pattern of the complex with two amphiphiles per metal center for one heating and cooling cycle. The peak at $q=0.16 \AA^{-1}$, which is continuous over the entire temperature range is associated with the $\mathrm{K}$-fluorescence of the $\mathrm{Fe}^{2+}$ ions at $E=6.4 \mathrm{keV}$. The two signals at $q=0.22 \AA^{-1}$ and $q=0.44 \AA^{-1}$ at $303 \mathrm{~K}$ represent the first and second order scattering peak suggesting a stacks-of-layers superstructure with a translational period of $d=29 \AA .{ }^{28}$ A peak-width analysis reveals a correlation length of coherent scattering layers of about $250 \AA$ corresponding to 8-9 single layers. At $328 \mathrm{~K}$, the two scattering peaks shift towards lower $q$ values of $q=0.206 \AA^{-1}$ and of low intensity at $q=0.41 \AA^{-1}$, respectively. This reflects a widening of the superstructure to $d=31 \AA$. The structural phase transition coincides with the melting of the amphiphilic molecules. ${ }^{34,35} \mathrm{At}$ the spin crossover temperature of $T_{\mathrm{SC}}=405 \mathrm{~K}$ a new scattering peak at $q=0.28 \AA^{-1}$ appears corresponding to a characteristic length of $d=22 \AA$. Additionally, a further scattering peak appears at $q=0.62 \AA^{-1}$ reflecting a structure with a characteristic length of $d \simeq 10 \AA$. The nature of this new structure is under further investigation. At temperatures around $415 \mathrm{~K}$, a weak peak $\left(q \sim 0.55 \AA^{-1}\right)$ becomes visible reflecting the diffuse scattering associated with the disorder induced during the spin crossover. Again, the resulting structural phase remains during cooling down to room temperature. This result verifies the assumption that the spin crossover is associated with the formation of the new structure.

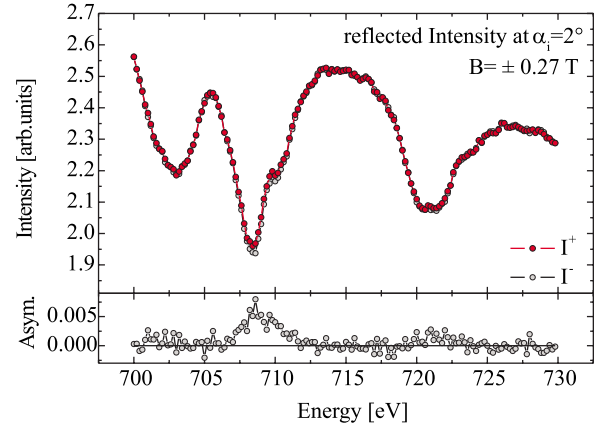

FIG. 5. (Color online) X-ray magnetic circular dichroism (XMCD) data at the $L_{3,2}$ edges of iron (top) of a PAC sample with two amphiphiles per metal center. The spectra is recorded in reflection at a fixed angle of incidence of $\alpha_{i}=2^{\circ}$. Bottom: The asymmetry ratio $A(E)=\left(I^{+}-I^{-}\right) /\left(I^{+}+I^{-}\right)$between both directions of the applied longitudinal field of $B= \pm 0.27 \mathrm{~T}$ demonstrates the occurrence of molecular magnetism.

In order to confirm our hypothesis that the spin crossover is induced by the structural phase transition of the amphiphilic matrix, PAC is prepared with $\mathrm{Ni}^{2+}$ as central ion. While $\mathrm{Ni}^{2+}-\mathrm{PAC}$ is isostructural to $\mathrm{Fe}^{2+}-\mathrm{PAC}$, it does not show any spin transition. The magnetic moment of $\mathrm{Ni}^{2+}-\mathrm{PAC}$ is measured for the same heating and cooling cycle described above. The observed magnetic moment of $\mathrm{Ni}^{2+}$-PAC is estimated to be $\mu_{\text {eff }}=3.14 \pm 0.05 \mu_{B} / \mathrm{Ni}^{2+}$, which is in reasonable agreement with literature values, e.g., with values obtained for supramolecular $[2 \times 2] \mathrm{Ni}^{2+}$ grids. ${ }^{29}$ The structural characterization was performed in the same way as described for the $\mathrm{Fe}^{2+}$-PACs. The $\mathrm{Ni}^{2+}$-PACs show also structural reorganization at the same temperatures as found for the $\mathrm{Fe}^{2+}$-PACs. Therefore, the structural phase transition of the amphiphilic matrix embedding the metal center in PAC is not affected by the transition metal ion.

\section{Soft x-ray magnetic circular dichroism}

To explore the origin of the magnetization in more detail, we applied soft $\mathrm{x}$-ray resonant scattering to these metallosupramolecular architectures. Because of the high absorption of the samples, measurements of the soft x-ray magnetic circular dichroism (XMCD) in transmission are not suitable. Therefore, the $\mathrm{X}$-ray reflectivity of the sample, placed on a silicon wafer, is measured at a fixed angle of incidence $\left(\alpha_{i}\right.$ $=2^{\circ}$ ) using circularly polarized synchrotron radiation and an alternating external field of $B= \pm 0.27 \mathrm{~T}$. This method offers high accuracy and is an element sensitive probe for the magnetism of the $\mathrm{Fe}^{2+}$ ions. For further details of the method, we refer to Mertins et al. ${ }^{30}$ and Zak et al. ${ }^{31}$ The measurements are performed at the UE56/1-PGM1 beam line at BESSY II using the ALICE diffractometer. ${ }^{32}$ Figure 5 shows a XMCD measurement of the complex containing two amphiphiles per metal center. The two distinct minima at 708 and $721 \mathrm{eV}$ correspond to the $L_{3}$ and $L_{2}$ absorption edges of iron, respectively. The intensities of the minima differ for both directions of the applied longitudinal field of $B= \pm 0.27 \mathrm{~T}$. The difference in reflectance is displayed by the asymmetry ratio 


$$
A(E)=\left(I^{+}-I^{-}\right) /\left(I^{+}+I^{-}\right) .
$$

A nonzero asymmetry ratio at the resonances is a clear evidence of a magnetization located at the investigated ion. We find differences in reflectance at the iron absorption edge of $2 \% \pm 0.2 \%$ for the 1:6-complex and $0.8 \% \pm 0.2 \%$ for the complex containing two amphiphiles per metal center. Therefore, we conclude that the magnetization of PAC is originating from the $\mathrm{Fe}^{2+}$ ions.

To gain further insight into the magnetic structure of PAC, we modeled the energy-dependent reflectance for circular polarized light as well as the asymmetries within the magneto-optical matrix formalism developed by Zak et al. ${ }^{31}$ Generally, the refractive index for $\mathrm{x}$ rays

$$
n_{ \pm}=1-(\delta \pm \Delta \delta)+i(\beta \pm \Delta \beta)
$$

is a complex quantity and both nonmagnetic (dispersion $\delta$, absorption $\beta)$ and magnetic $(\Delta \delta, \Delta \beta)$ terms of the refractive index contribute to the reflected signal. As input for the calculations, we use the functions $\delta(E), \beta(E)$ and $\Delta \delta(E), \Delta \beta(E)$ of bulk iron in the vicinity of the $L_{3,2}$ absorption edges. ${ }^{33}$ Therefore, only a qualitative result of the order of magnitude of the magnetic moment carried by the $\mathrm{Fe}^{2+}$ ions can be given. To simulate the scattering profile, we use the same stack-of-layer superstructure with a translational period of $d=29 \AA$ as observed by small angle x-ray scattering. This formalism yields a magnetic moment of $5 \pm 4 \mu_{B} / \mathrm{Fe}^{2+}$, which is in agreement with the value observed by magnetic measurements and is, therefore, a proof of the localization of the magnetic moment within the MEPE layers.

\section{CONCLUSION}

In conclusion, we show that a fine-tuning of spin crossover phenomenon can be achieved through the design of the supramolecular architecture. The modularity of this approach provides extensive control of magnetic properties by adjustment of the self-assembly conditions. We present significant evidence that the spin crossover in PACs is induced by a structural phase transition in the amphiphilic matrix. The temperature as well as the temperature range at which the spin crossover occurs depends on the ratio of metal centers to amphiphilic molecules. We investigate spin crossover temperatures depending on the ratio of amphiphiles per metal center to be $410 \mathrm{~K}$ for the 1:2-system, $425 \mathrm{~K}$ for the $1: 4$ system, and $452 \mathrm{~K}$ for the 1:6-system. The final magnetic moment of $4.8 \mu_{B} / \mathrm{Fe}^{2+}$ indicates that close to all metal ions participate in the spin crossover. In contrast to thin films, the magnetization remains stable during cooling in the bulk phase. Temperature resolved small angle x-ray scattering measurements reveal a structural change at the same temperature at which the spin crossover occurs. Like the highspin state, this new structural phase remains stable during cooling. These findings strongly support our model of a structurally induced and stabilized high-spin state in these systems. Apparently, the alkyl chains do not easily reorganize upon cooling so there is no annealing of defects in the metallopolyelectrolyte as well. The different magnetic behavior of bulk and thin films might also be of structural origin. Whereas in thin films the PACs are organized in preferentially oriented two dimensional planes, the bulk phase is most likely composed of randomly oriented domains, which inhibit relaxation upon cooling. Our results illustrate that an adjustment of spin crossover properties can be achieved through the design of the supramolecular architecture by deliberately adjusting the self-assembly conditions and therefore represent an approach to a selective implementation of magnetic properties into supramolecular materials.

\section{ACKNOWLEDGMENTS}

This work was supported by Deutsche Forschungsgemeinschaft as part of the priority program 1137 "Molecular Magnetism." Resonant soft x-ray scattering was supported by the German Federal Ministry of Education and Research (BMBF) under Grant No. 03ZA6BC2 (Alice Diffractometer).
${ }^{1}$ J.-M. Lehn, Science 295, 2400 (2002).

${ }^{2}$ G. M. Whitesides and B. Grzybowski, Science 295, 2418 (2002).

${ }^{3}$ G. J. Halder, C. J. Kepert, B. Moubaraki, K. S. Murray, and J. D. Cashion, Science 298, 1762 (2002).

${ }^{4}$ J.-M. Lehn, Proc. Natl. Acad. Sci. U.S.A. 99, 4762 (2002).

${ }^{5}$ O. Kahn and C. J. Martinez, Science 279, 44 (1998).

${ }^{6}$ D. Gatteschi, A. Caneschi, L. Pardi, and R. Sessoli, Science 265, 1054 (1994).

${ }^{7}$ D. Gatteschi and R. Sessoli, J. Magn. Magn. Mater. 272-276, 1030 (2004).

${ }^{8}$ P. Gütlich and H. A. Goodwin, Top. Curr. Chem. 233, 49 (2004) and literature cited herein.

${ }^{9}$ J. Rojo, J.-M. Lehn, G. Baum, D. Fenske, O. Waldmann, and P. Müller, Eur. J. Inorg. Chem. 3, 517 (1999).

${ }^{10}$ E. C. Constable et al., Chem.-Eur. J. 5, 498 (1999).

${ }^{11}$ Y. Galyametdinov, V. Ksenofontov, A. Prosvirin, I. Ovchinnikov,
G. Ivanova, P. Gütlich, and W. Haase, Angew. Chem., Int. Ed. 40, 4269 (2001).

${ }^{12}$ H. Soyer, E. Dupart, C. J. Gomez-Garcia, C. Mingotaud, and P. Delhaes, Adv. Mater. (Weinheim, Ger.) 11, 382 (1999).

${ }^{13}$ E. Breuning, M. Ruben, J.-M. Lehn, F. Renz, Y. Garcia, V. Ksenofontov, P. Gütlich, E. Wegelius, and K. Rissanen, Angew. Chem., Int. Ed. 39, 2504 (2000).

${ }^{14}$ M. Ruben, E. Breuning, J.-M. Lehn, V. Ksenofontov, F. Renz, P. Gütlich, and G. B. M. Vaughan, Chem.-Eur. J. 9, 4422 (2003).

${ }^{15}$ O. Waldmann, M. Ruben, U. Ziener, P. Müller, and J.-M. Lehn, Inorg. Chem. 45, 6535 (2002).

${ }^{16}$ Y. Bodenthin, U. Pietsch, H. Möhwald, and D. G. Kurth, J. Am. Chem. Soc. 127, 3110 (2005).

${ }^{17}$ P. Gütlich, Y. Garcia, P. J. van Koningsbruggen, and F. Renz, in Introduction to Physical Techniques in Molecular Magnetism: Structural and Macroscopic Techniques, edited by F. Palacio, E. 
Ressouche, and J. Schweizer (University Press, Zaragoza, Spain, 2001), p. 321.

${ }^{18}$ D. Chernyshov, H. B. Bürgi, M. Hostettler, and K. W. Törnroos, Phys. Rev. B 70, 094116 (2004).

${ }^{19}$ S. Hayami, T. Kawahara, G. Juhasz, K. Kawamura, K. Uehashi, O. Sato, and Y. Maeda, J. Radioanal. Nucl. Chem. 255, 443 (2003).

${ }^{20}$ C. Cantin, J. Kliava, A. Marbeuf, and D. Mikailitchenko, Eur. Phys. J. B 12, 525 (1999).

${ }^{21}$ C. P. Slichter and H. G. Drickamer, J. Chem. Phys. 56, 2142 (1972).

${ }^{22}$ D. G. Kurth, P. Lehmann, and M. Schütte, Proc. Natl. Acad. Sci. U.S.A. 97, 5704 (2000).

${ }^{23}$ A. Meister, G. Förster, A. F. Thünemann, and D. Kurth, ChemPhysChem 4, 1095 (2003).

${ }^{24}$ L. Merz and W. Haase, J. Chem. Soc. Dalton Trans. 1980, 875 (1980).

${ }^{25}$ H. Luecken, Magnetochemie, Teubner Studienbücher Chemie BG (Teubner Verlag, Stuttgart-Leipzig, 1999).

${ }^{26}$ U. Pietsch, J. Grenzer, T. Geue, F. Neissendorfer, G. Brezsesinski, C. Symietz, H. Möhwald, and W. Gudat, Nucl. Instrum. Methods Phys. Res. A 467, 1077 (2001).

${ }^{27}$ Y. Bodenthin, J. Grenzer, R. Lauter, U. Pietsch, P. Lehmann, D.
G. Kurth, and H. Möhwald, J. Synchrotron Radiat. 9, 206 (2002).

${ }^{28}$ D. G. Kurth, A. Meister, A. F. Thünemann, and G. Förster, Langmuir 19, 4055 (2003).

${ }^{29}$ O. Waldmann, J. Hassmann, P. Müller, D. Volkmer, U. S. Schubert, and J. M. Lehn, Phys. Rev. B 58, 3277 (1998).

${ }^{30}$ H.-C. Mertins, D. Abramsohn, A. Gaupp, F. Schäfers, W. Gudat, O. Zaharko, H. Grimmer, and P. M. Oppeneer, Phys. Rev. B 66, 184404 (2002).

${ }^{31}$ J. Zak, E. R. Moog, C. Liu, and S. D. Bader, Phys. Rev. B 43, 6423 (1991).

${ }^{32}$ J. Grabis, A. Nefedov, and H. Zabel, Rev. Sci. Instrum. 74, 4048 (2003).

${ }^{33}$ C. T. Chen, Y. U. Idzerda, H.-J. Lin, N. V. Smith, G. Meigs, E. Chaban, G. H. Ho, E. Pellegrin, and F. Sette, Phys. Rev. Lett. 75, 152 (1995).

${ }^{34}$ Y. Bodenthin, U. Pietsch, J. Grenzer, T. Geue, H. Möhwald, and D. G. Kurth, J. Phys. Chem. B 109, 12795 (2005).

${ }^{35}$ The alkyl chain packing of DHP gives rise to scattering peaks at $q \sim 1.5 \AA^{-1}$. A third detector measuring the wide angle scattering was placed at an angle of $2 \theta=28.6^{\circ}$ simultaneously. The observed WAXS peak vanishes above $328 \mathrm{~K}$. For further details, please refer to Ref. 34 . 